\title{
Liver abscess caused by Salmonella choleraesuis
}

This article was published in the following Dove Press journal:

International Journal of General Medicine

20 April 2015

Number of times this article has been viewed

\author{
Takashi Kamatani' \\ Takemichi Okada ${ }^{2}$ \\ Hiroyoshi Iguchi \\ Yoshihito Takahashi ${ }^{3}$ \\ Hiroaki Yokomori' \\ 'Department of Internal \\ Medicine, ${ }^{2}$ Department of \\ Radiology, ${ }^{3}$ Department of \\ Surgery, Kitasato University \\ Medical Center, Saitama, Japan
}

Correspondence: Hiroaki Yokomori Kitasato University Medical Center, 6-I00 Arai, Kitamoto-shi, Saitama 364-850I, Japan

$\mathrm{Tel}+8 \mid 48593$ I2I 2

Fax $+8 \mid 48593$ I239

Email yokomori@insti.kitasato-u.ac.jp
Abstract: A 65-year-old man with long-term alcohol abuse presented with intermittent fever. Abdominal computed tomography revealed multiple masses. Abscess blood and pus cultures conducted after percutaneous catheter drainage with pigtail catheters yielded Salmonella choleraesuis. Antibiotic treatment with meropenem was started using multiple catheters in the liver. Drainage catheters in different locations were exchanged several times with larger-bored catheters. After septicemia was detected, abscesses spread to the peritoneal cavity. Pleural complications developed. Antibiotic treatment, with careful drainage guided by ultrasound or computed tomography, controlled the abscesses and complications. This report describes the difficult clinical course and treatment of a liver abscess from $S$. choleraesuis.

Keywords: liver abscess, Salmonella choleraesuis, bacteremia, CT

\section{Introduction}

Pyogenic abscesses of the liver are most commonly found in the right lobe, followed by the left and caudate lobes. Escherichia coli and Klebsiella pneumoniae are the most common isolates followed by Bacteroides, Streptococcus and Enterococcus species. ${ }^{1,2}$ In particular, E. coli is cultured most frequently in Western countries ${ }^{1}$ and Klebsiella in Asian countries. ${ }^{2}$

Salmonella infection is a common bacterial disease that affects the intestinal tract. The bacteria are typically carried in animal and human intestines and are shed through feces. Salmonella is ubiquitous and is a worldwide public health problem. Diseases caused by nontyphoidal salmonellae varied from mild self-limited gastroenteritis to severe, invasive infections such as bacteremia, osteomyelitis, and meningitis. ${ }^{3}$ Hepatic abscesses secondary to nontyphoidal salmonellosis are very rare in the general population, especially in developed countries. ${ }^{3}$ This report describes a case of liver abscess caused by Salmonella choleraesuis with a difficult to predict clinical course which was difficult to treat.

\section{Case presentation}

A 65-year-old man presented at our hospital complaining of intermittent fever and chills for 14 days. He denied any recent foreign travel, and had no other constitutional or abdominal symptoms, but reported anorexia and generalized fatigue for 1 month. He had a continuous history of alcohol consumption of 150-180 g (alcohol conversion) daily for 40 years. He denied any history of systemic diseases including diabetes mellitus, hypertension, and cirrhosis. He had no history of non-recent travel, exposure to animals, or invasive medical procedures. Physical examination showed remarkable 
generalized muscle wasting of the whole body, and mild right upper quadrant and epigastric tenderness. His body temperature was $40^{\circ} \mathrm{C}$. Pulmonary, cardiac and abdominal examinations revealed no abnormalities. An electrocardiogram was normal. The hematological tests showed white blood cell count of 14.13/ $\mathrm{L}$ (normal range 3.30-9.00), hemoglobin $14.5 \mathrm{~g} / \mathrm{dL}$ (normal range 11.5-15.0), and platelet count $20.7 \times 10^{4} / \mu \mathrm{L}$ (normal range 14-34). Prothrombin time (international normalized ratio) was $12.1 \mathrm{~s}(93 \%)$ (normal range 9.5-12.0 s). Biochemical tests revealed total bilirubin of $1.6 \mathrm{mg} / \mathrm{dL}$ (normal range $0.2-1.0$ ), direct bilirubin $0.3 \mathrm{mg} / \mathrm{dL}$ (normal range $0.0-0.2$ ), aspartate aminotransferase $65 \mathrm{IU} / \mathrm{L}$ (normal range 10-35), alanine aminotransferase 77 IU/L (normal range 5-40), alkaline phosphatase $597 \mathrm{IU} / \mathrm{L}$ (normal range 115-359), $\gamma$-guanosine triphosphate $367 \mathrm{IU} / \mathrm{L}$ (normal range $<80$ ), and C-reactive protein $25.16 \mathrm{mg} / \mathrm{dL}$ (normal value $<0.5$ ). Tests for hepatitis B virus (HBV), hepatitis C virus (HCV) and human immunodeficiency virus (HIV) were negative. Blood culture yielded $S$. choleraesuis. A chest X-ray showed an elevated right hemidiaphragm. Abdominal ultrasonography findings showed multiple low density lesions with sizes ranging $2.3-4.1 \mathrm{~cm}$ in the right and left lobes of the liver.

Computed tomography (CT) scan of the abdomen revealed multiple masses in various segments (mainly segments IV, VI, VII and VIII) of the right hepatic lobe, and segment II of left hepatic lobe. The masses ranged in size from $1 \mathrm{~cm}$ to $3 \mathrm{~cm}$, and were hypodense with irregular margins, strongly suggestive of liver abscesses (Figure 1A-F). Moreover, CT imaging showed a thick, hyper-enhanced wall and adjacent fat stranding in the appendix (Figure $1 \mathrm{G})$. Antibiotic treatment with cefoperazone was initiated, but was switched to meropenem on the patient's fourth day in hospital when his fever did not subside. In addition, ultrasound-guided percutaneous catheter drainage (PCD) was performed for rapidly expanding hepatic abscesses measuring over $4 \mathrm{~cm}$. Two 8 Fr pigtail catheters placed in segments VI and VIII yielded thick tenacious pus (Figure 2A and B). Diagnostic aspiration of the lesion yielded $50 \mathrm{~mL}$ of purulent material, which grew $S$. choleraesuis [07:c:1,5; $\mathrm{H}_{2} \mathrm{~S}(-)$, invA (+), stn (+)], Streptococcus milleri, Enterobacteriaceae, and Bacteroides. Auto-drainage from each catheter yielded approximately $20 \mathrm{~mL}$ per day. The cavity was washed with sterile saline solution injected gently to avoid pressure. On the patient's fourteenth day in hospital, drainage fluid decreased suddenly, but body temperature remained elevated. Epigastric tenderness aggravated and bowel sounds decreased. The abdomen was soft, and no
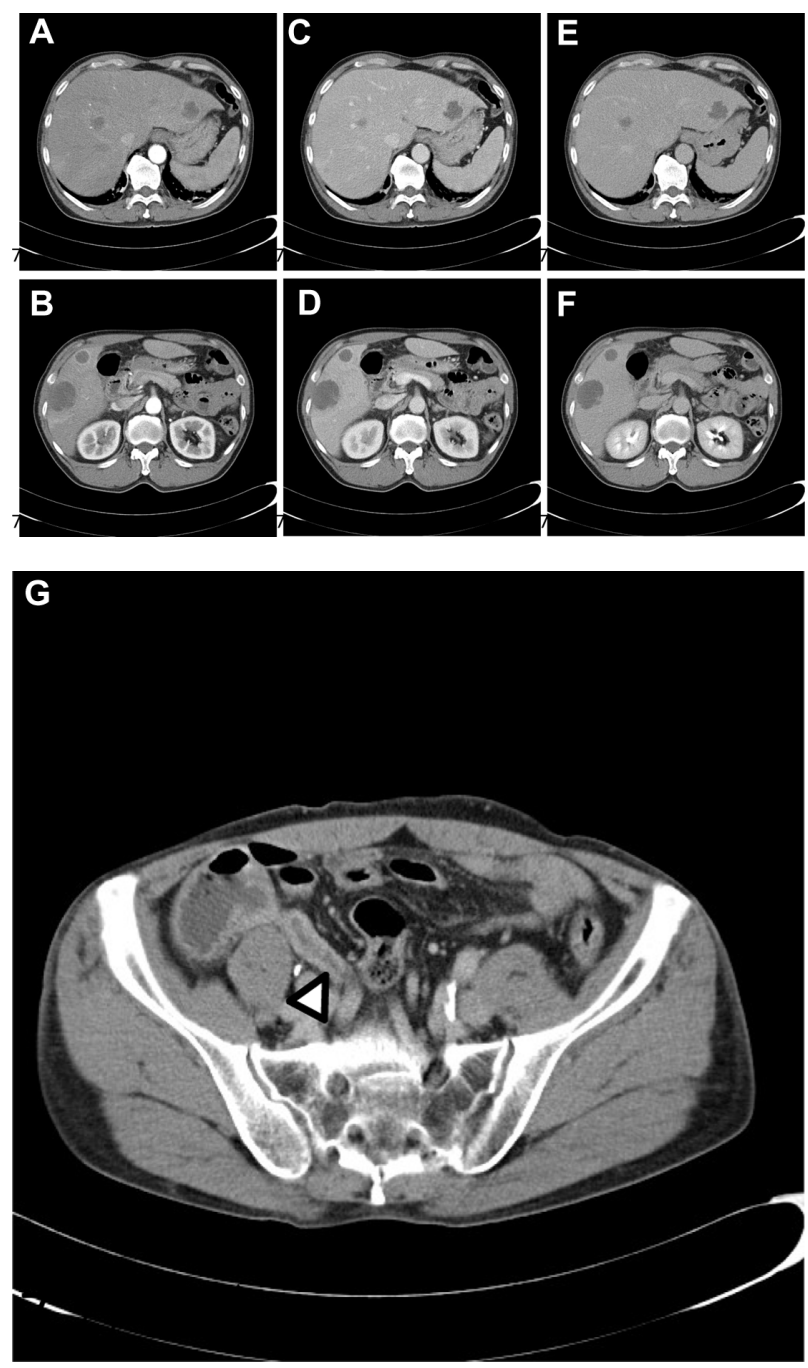

Figure I Abdominal CT findings on admission.

Notes: Plain CT scans show hypodense masses with poorly defined margin in the periphery of right anterior and posterior segments of liver ( $\mathbf{A}$ and $\mathbf{B})$. Arterial phase (30 seconds) dynamic contrast-enhanced CT scans show double-target sign consisting of a central cystic area with an inner enhanced rim and an outer hypodense zone in anterior and posterior segments of right lobe, and also left lobe (C and D). Portal phase ( 90 seconds) dynamic CT scans show thick rim enhancement (E and $\mathbf{F}$ ). Late phase dynamic contrast-enhanced CT scan of the pelvic region shows a thick hyper-enhanced wall in the appendix $(\mathbf{G})$. Arrow head denotes appendix.

Abbreviation: CT, computed tomography.

muscle guarding and rebound tenderness were found. An abdominal CT scan showed further spread of abscesses in segments II, IV, VI, VII and VIII of the liver and in the subphrenic space (Figure 2A and B). Since fever did not resolve, transcatheter regional hepatic arterial infusion of antibiotics was performed. Because of peritonitis, abdominal CT was performed and revealed air-containing abscesses in the right and left subphrenic regions. Then, right pleural effusion and empyema (Figure 2C) with clinical features of respiratory failure appeared after the twentieth day. His clinical condition decompensated quickly thereafter, requiring endotracheal intubation and mechanical ventilation on the patient's 

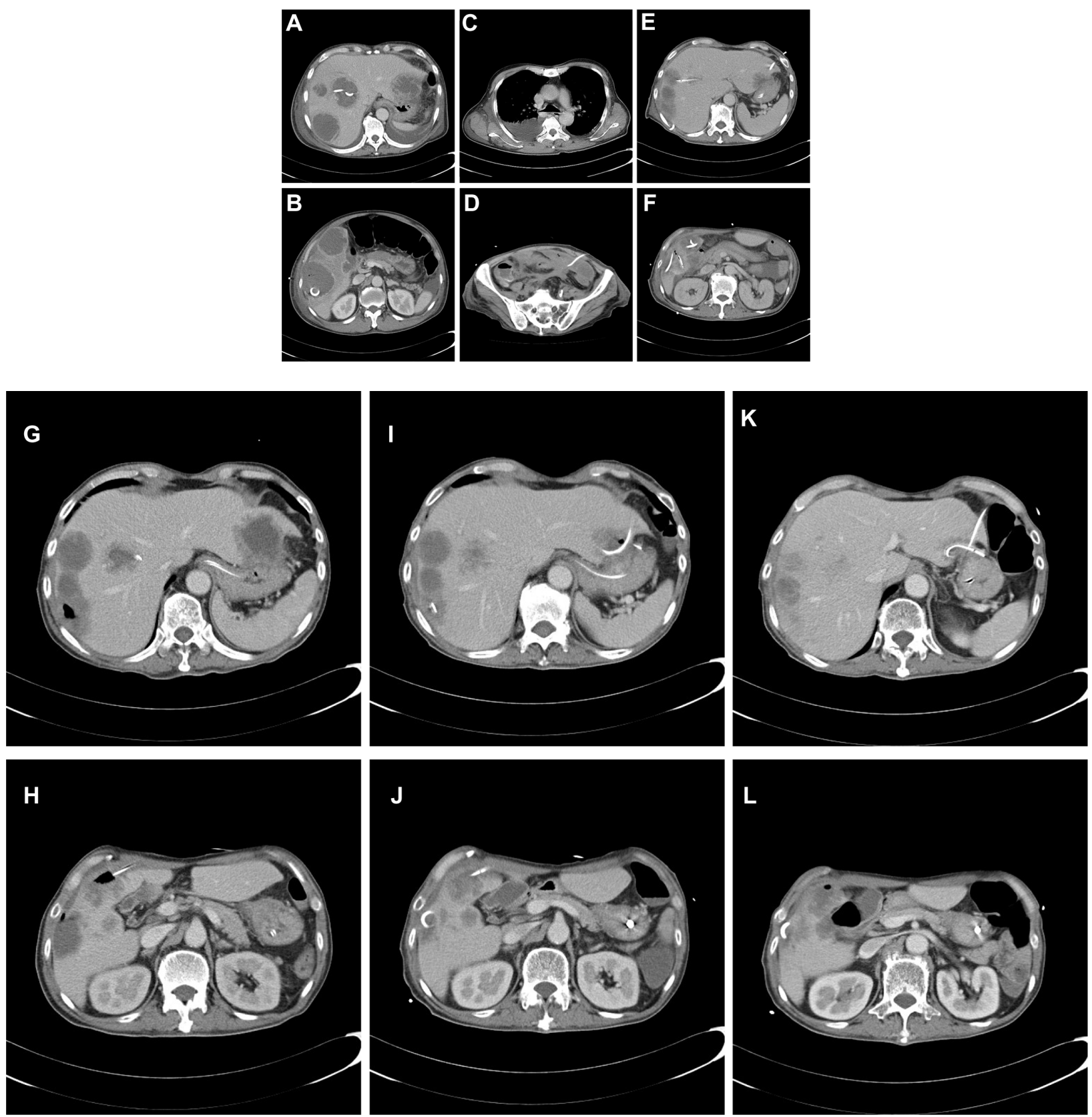

Figure 2 The course of liver abscess depicted on CT.

Notes: $8 \mathrm{Fr}$ pigtail catheters for percutaneous drainage are placed in segments VI and VIII (A and B). Right pleural effusion and empyema are evident (C). 8 Fr pigtail catheters have been changed to $12 \mathrm{Fr}$ or $14 \mathrm{Fr}$ large-bored catheters, and a $8 \mathrm{Fr}$ pigtail catheter is placed in segmental VII (D and E). An intraperitoneal abscess is observed (F). Percutaneous catheter drainage has been removed from segment II after the patient's clinical condition and laboratory test results improved (G and $\mathbf{H}$ ). Percutaneous catheter drainage is restarted to drain hepatic abscesses; 12 or I4 Fr large-bored catheters for segments IV, VI and VII, and 8F-pigtail catheters for segments II and VIII ( $\mathbf{I}$ and $\mathbf{J})$. CT scans show hypodense mass with well-defined margin at periphery of right anterior and posterior segment, and left segment of liver (K and $\mathbf{L})$. These findings show resolved abscess formation. The abscesses seen at admission have resolved. Abbreviation: CT, computed tomography.

twenty-fifth day in hospital. Due to incomplete drainage by catheters, CT guided percutaneous needle aspiration of other abscesses was performed. Antibiotic treatment with meropenem was continued, and a few blood transfusions were performed. The patient was clinically improved, but abscesses persisted despite adequate catheter placement. The $8 \mathrm{Fr}$ PCD catheters were exchanged to $12 \mathrm{Fr}$ or $14 \mathrm{Fr}$ large- bored catheters and placed into the abscess cavity. An additional 8 Fr pigtail catheter was placed into segmental VII on the patient's thirtieth day in hospital (Figure 2D and E). A colobiliary fistula with a liver abscess was detected by PCD catheter (Figure 3). Surprisingly, on the patient's thirtieth day in hospital, an intraperitoneal abscess was noted and $30 \mathrm{~mL}$ of turbid pus was aspirated from the peritoneal 


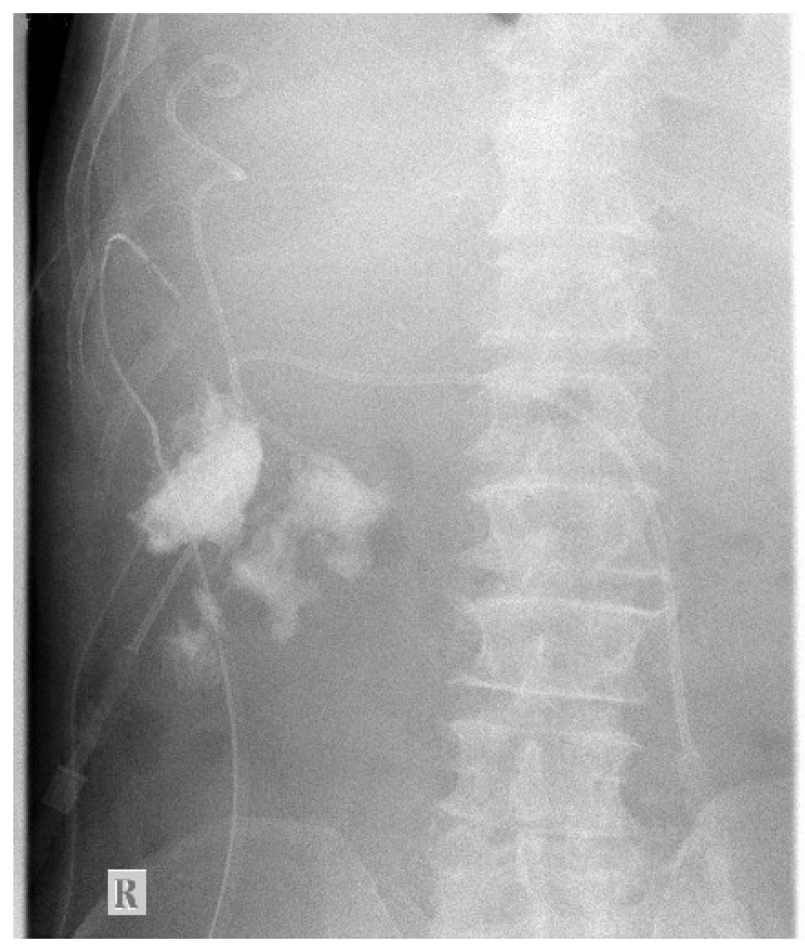

Figure 3 Abscessogram of segmental VI.

Note: A colobiliary fistula is visible. $R$ denotes the right side.

cavity (Figure 2F). Thereafter his clinical condition and laboratory data improved gradually. We removed PCD from segment II. However, CT scans showed gradual enlargement of abscesses in segments II, VII and VIII of the liver (Figure 2G-J) on days 40-50. PCD catheters were reinserted into segments II and VII, and the catheter in segment VIII was exchanged. Upon aspiration of the lesions, purulent material yielded Enterococcus faecium from segment II in the left hepatic lobe. The E. faecium isolate was resistant to $\beta$-lactams and carbapenem. The oxazolidinone derivative linezolid was administered. Moreover, Stenotrophomonas maltophilia and Candida albicans were detected from cloudy materials aspirated from lesions in segments II and VII on the patient's ninetieth day in hospital. Trimethoprimsulfamethoxazole (TMT-SMXD) and fosfluconazole (INN) were administered. Abdominal CT repeated on the patient's one-hundredth day in hospital showed resolution of the previously described masses (Figure $2 \mathrm{~K}$ and $\mathrm{L}$ ) and the patient's clinical condition improved gradually (Figure 4).

\section{Discussion}

The severity of pyogenic abscess depends on the bacterial source and the underlying condition of the patient. ${ }^{1,2}$ The present case was a patient with liver abscesses accompanied by a peritoneal abscess and septicemia caused by S. choleraesuis, which had a unpredictable clinical course and was difficult to treatment.

The presence of multiple abscess cavities does not preclude the use of PCD, but does necessitate placement of several catheters. ${ }^{1}$ In the present case, we performed several PCD catheter exchanges. We initially used only 8 Fr pigtail catheters, which may have been too narrow to drain the thick pus. Although highly successful, PCD fails in approximately $10 \%$ cases of pyogenic abscesses. ${ }^{2}$ Operative intervention remains the second-line therapy, although surgical treatment should be considered when clinically indicated and in patients with fungal infection. ${ }^{4}$ Hope et $\mathrm{al}^{5}$ stratified 107 patients with pyogenic liver abscess into 3 types: I (small, $<3 \mathrm{~cm}$ ); II (large, $>3 \mathrm{~cm}$, unilocular), and III (large, $>3 \mathrm{~cm}$, complex multilocular). They proposed a treatment algorithm with small abscesses treated with antibiotics alone; large, uniloculated abscesses treated with percutaneous drainage and antibiotics; and large, multiloculated abscesses treated with surgical therapy. In our case, treatment with PCD and antibiotics was successful. Surgical salvage is associated with a high mortality. We were not able to perform surgical open drainage because the patient was already in a consumed condition.

Moreover, this patient also had concurrent bacteremia. Mortality in pyogenic liver abscesses is associated with older age as well as comorbidities such as bacteremia, cirrhosis, chronic renal failure, and cancer. ${ }^{6}$ A CT scan showed abscesses in the subphrenic space, which may have arisen from rupture of liver abscesses. In the past, surgical intervention to drain the abscess and clean the abdominal cavity was the only means of saving the lives of patients with ruptured pyogenic liver abscesses. ${ }^{7}$ The present case had multiple liver abscesses, intraperitoneal abscesses, and bacteremia caused by S. choleraesuis. Previously, all reported Salmonella hepatic abscess cases were treated with antibiotics and either surgical or percutaneous catheter drainage. ${ }^{8,9}$

However, in a case report of solitary hepatic abscess caused by Salmonella, antibiotics alone resulted in complete clinical recovery. ${ }^{8,9}$

The prevalence of liver abscesses in cirrhosis is, constituting only $0.9 \%-13.0 \%$ of all cirrhosis cases. ${ }^{1,2,10}$ Among incidence and fatality rate, alcoholic cirrhosis was common. ${ }^{11}$

In our case, a CT scan showed a large hepatic abscess containing gas. The destruction of the hepatic parenchyma in a "gas-forming" liver abscess is more severe than in a "non-gas-forming" abscess. ${ }^{12}$ Presumably the hepatocolic fistula in the present patient was a rare complication, and it 


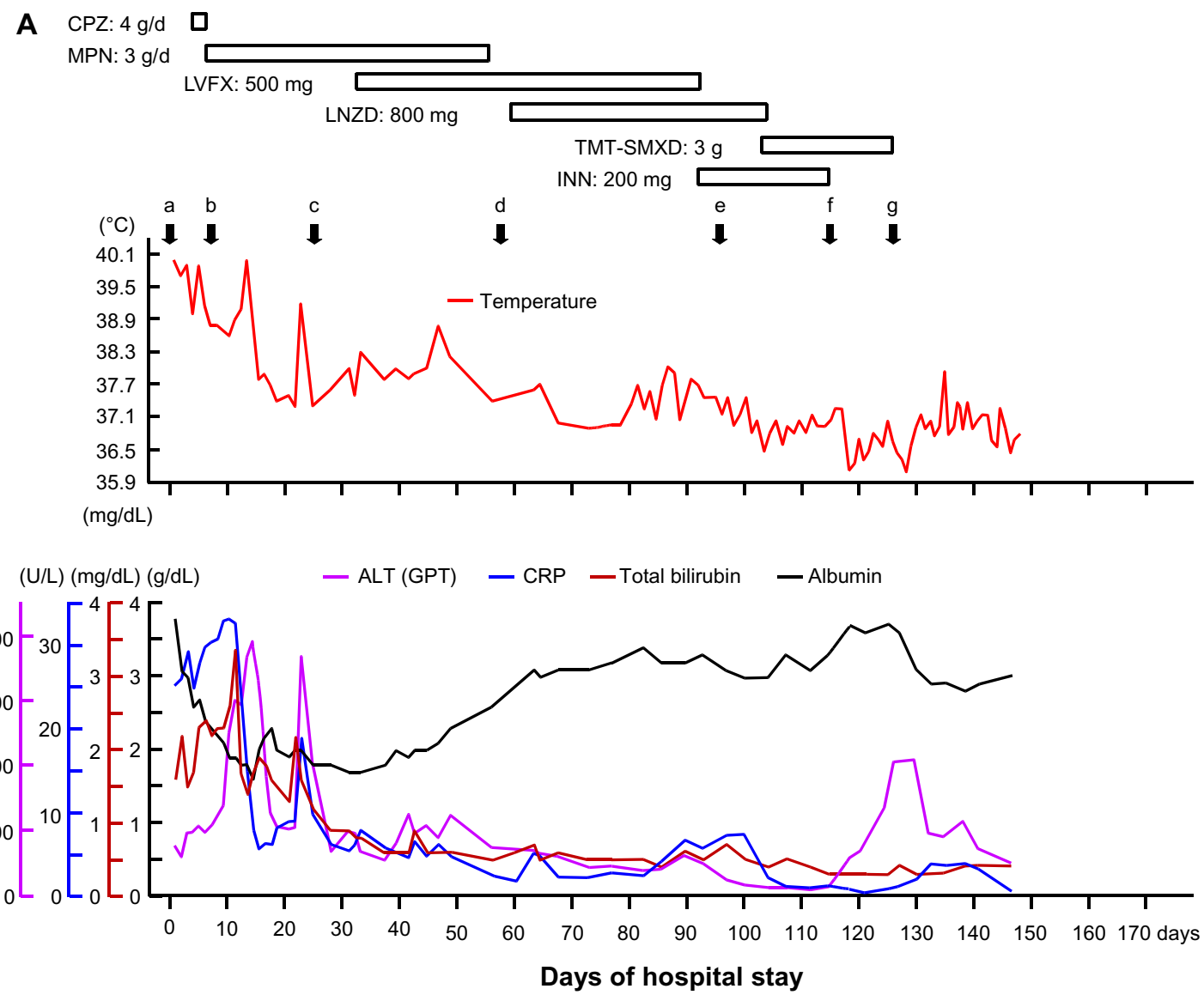

B a

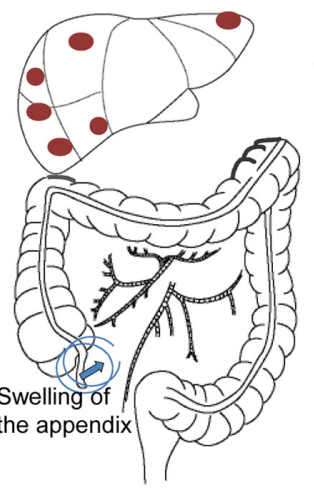

b

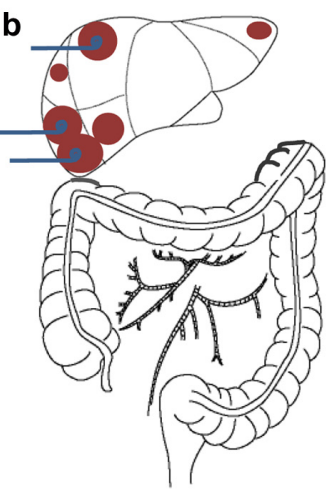

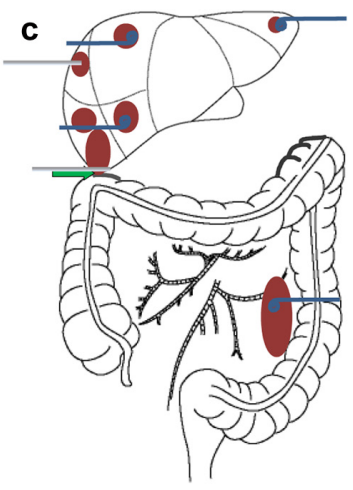

d.

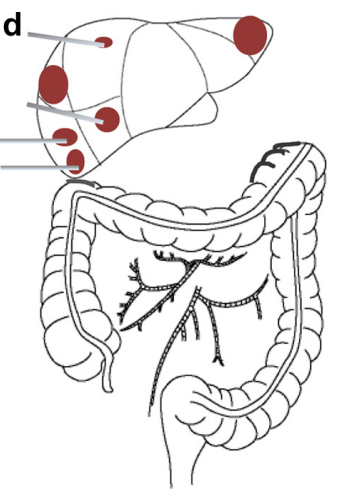

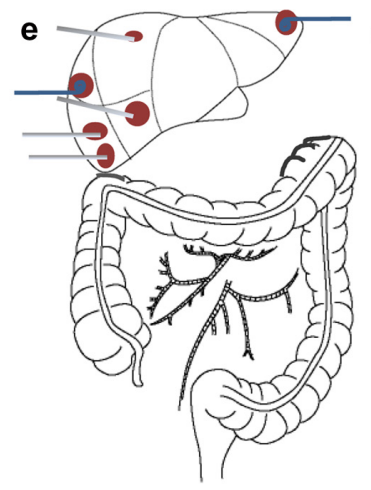

$$
\text { f }
$$

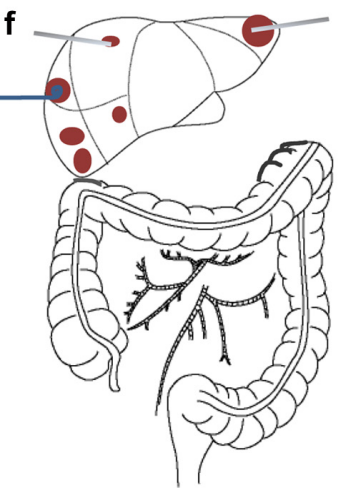

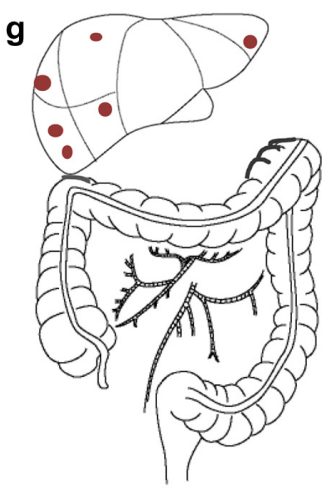

8 Fr-pigtail

14 or $12 \mathrm{Fr}$ large-bored catheter

$\Longrightarrow$ Colobiliary fistula

Figure 4 Clinical course (A) and diagrams of percutaneous drainage (B).

Abbreviations: CPZ, cefoperazone; MPN, meropenem; LVFX, levofloxacin; LNZD, linezolid; TMT-SMX, trimethoprim-sulfamethoxazole; INN, fosfluconazole; ALT (GPT), alanine aminotransferase (glutamic pyruvic transaminase); CRP, C-reactive protein. 
may have been related to the more destructive nature of the gas-forming pyogenic liver abscess.

On admission, a CT scan showed a thick hyper-enhanced wall and adjacent fat stranding in the appendix. Due to the invasive nature of $S$. choleraesuis, large numbers of the bacteria have been shown to be present in macrophages in the enteric mucosa and in related lymph nodes. ${ }^{13-16}$ Because Salmonella can affect the regional mesenteric lymph nodes, adenitis and terminal ileitis may occur, which mimics acute appendicitis. ${ }^{17} \mathrm{~S}$. choleraesuis is a prominent cause of invasive infections. Among nontyphoidal salmonellae, S. choleraesuis has the highest invasion index, which is the number of extraintestinal isolates divided by the total number of isolates. ${ }^{13}$ This species is highly pathogenic to humans, usually causing septicemia disease with little involvement of the intestinal tract. In an animal model, $S$. choleraesuis colonizes and invades the intestinal epithelium, disseminates to peripheral organs, and causes septicemia. ${ }^{15}$ Salmonellae carried by asymptomatic swine may serve as a source of Salmonella infection to humans via contamination of pork products. It was unclear whether the patient in this case had consumed contaminated raw pork. S. choleraesuis usually causes systemic infections in humans, who require antimicrobial treatment. A recent retrospective analysis of adult patients with serotype choleraesuis bacteremia showed that most of the patients had obvious risk factors for salmonellosis, including malignancy, cirrhosis, systemic lupus erythematosus, and previous use of corticosteroids. ${ }^{18}$ It was notable that $21 \%$ of the bacteremic patients subsequently developed focal infections, including septic arthritis, pneumonia, peritonitis, and cutaneous abscess. ${ }^{18}$

Our patient had a continuous history of excessive alcohol consumption of $150 \mathrm{~g}$ per day for 40 years. Impaired immunity in patients with chronic alcohol use has long been described in the medical literature. ${ }^{19}$ A study suggests that diabetes mellitus and alcoholism are significant risk factors for developing metastatic infections from pyogenic liver abscesses. ${ }^{20}$ Taken together, alcoholism and the patient's muscle wasted condition may have predisposed the patient to developing hepatic abscesses by $S$. choleraesuis and the unpredictable clinical course of metastatic infections in the peritoneal cavity, blood and probably lung. Furthermore, the clinical course showed rapid accumulation of pleural effusion and empyema, probably as a result of the pressure effect of the liver abscess on the right hemi-diaphragm and the development of pleural metastasis from pyogenic liver abscesses. In animal models, chronic ingestion of ethanol decreased pulmonary glutathione concentration, increased alveolar barrier permeability, and increased the risk of acute lung injury. ${ }^{21,22}$ The present case and findings in animal studies above might explain, at least in part, why alcoholism tended to predispose to pleuropulmonary metastases from pyogenic liver abscesses.

Besides antibiotics, indwelling medical devices such as PCD and urinary catheters were used in this patient. Use of antibiotics, catheters and immunosuppression alter the usual host-microbe balance and facilitate infection by bacteria such as E. faecium that are resistant to broad spectrum $\beta$-lactams and other antibiotics. Linezolid is an oxazolidinone antibiotic that inhibits bacterial ribosomal protein synthesis ${ }^{23}$ and has been approved for the treatment of vancomycin-resistant enterococcus infections. Salvage therapy with linezolid might be a safe and efficacious treatment for E. faecium infection.

\section{Conclusion}

$S$. choleraesuis infection does not always present as gastroenteritis. This case of liver abscess caused by $S$. choleraesuis had an unpredictable clinical course and was difficult to treat. To our knowledge, this is the first report of liver abscess accompanied by peritoneal abscess and bacteremia caused by S. choleraesuis, which was successfully treated conservatively by parenteral antibiotics and ultrasound- and CT-guided drainage.

\section{Disclosure}

Hiroaki Yokomori received research grants from MSD (Merck, Sharp and Dohme, UK). The authors report no other conflicts of interest in this work.

\section{References}

1. Seeto R, Rockey DC. Pyogenic liver abscess: changes in etiology, management and outcome. Medicine. 1996;75:99-113.

2. Chou FF, Sheen-Chen SM, Chen YS, Chen MC. Single and multiple pyogenic liver abscesses: clinical course, etiology and results of treatment. World J Surg. 1997;21:384-389.

3. Feasey NA, G Dougan G, Kingsley RA, Heyderman RS, Gordon MA. Invasive non-typhoidal Salmonella disease: an emerging and neglected tropical disease in Africa. Lancet. 2012;379:2489-2499.

4. Mezhir JJ, Fong Y, Jacks LM, et al. Current management of pyogenic liver abscess: surgery is now second-line treatment. J Am Coll Surg. 2010;210:975-983

5. Hope WW, Vrochides DV, Newcomb WL, Mayo-Smith WW, Iannitti DA. Optimal treatment of hepatic abscess. Am Surg. 2008;74:178-182.

6. Meddings L, Myers RP, Hubbard J, et al. A population-based study of pyogenic liver abscesses in the United States: incidence, mortality, and temporal trends. Am J Gastroenterol. 2010;105:117-124.

7. Chou FF, Sheen-Chen SM, Lee TY. Rupture of pyogenic abscess. Am J Gastroenterol. 1995;90:767-770.

8. Tulachan SS, Wilkins CE, Cutrona AF, Gemmel D, Marnejon TP. Hepatic abscess associated with Salmonella serotype B in a chronic alcoholic patient. Hepatobiliary Pancreat Dis Int. 2013;12:440-442. 
9. Von Eberts EM. Abscess of the liver occurring in association with or following typhoid fever. Am J Med Sci. 1911;141:803-826.

10. Kuo CM, Kuo CH, Changchien CS. Liver abscess in patients with cirrhosis of the liver: a 12-year experience. Gastroenterology. 2001;36: 552-556.

11. Mølle I, Thulstrup AM, Vilstrup H, Sørensen HT. Increased risk and case fatality rate of pyogenic liver abscess in patients with liver cirrhosis: a nationwide study in Denmark. Gut. 2001;48:260-263.

12. Yang CC, Chen CY, Lin XZ, Chang TT, Shin JS, Lin CY. Pyogenic liver abscess in Taiwan: Emphasis on gas-forming liver abscess in diabetics. Am J Gastroenterol. 1993;88:1911-1915.

13. Chiu CH, Lin TY, Ou JT. Prevalence of the virulence plasmids of nontyphoid Salmonella in the serovars isolated from humans and their association with bacteremia. Microbiol Immunol. 1999;43:899-903.

14. Farmer JJ, Boatwright KD, Janda JM. Enterobacteriaceae: Introduction and Identification. In Manual of Clinical Microbiology. Murray PR, Jorgensen JH, Landry ML, Pfaller MA, editors, Ninth edition. ASM press, Washington, DC, USA. 2007:649-669.

15. Chiu CH, Su LH, Chu C. Salmonella enterica serotype Choleraesuis: epidemiology, pathogenesis, clinical disease, and treatment. Clin Microbiol Rev. 2004;17:311-322.

16. Pospischil A, Wood RL, Anderson TD. Peroxidase-antiperoxidase and immunogold labeling of Salmonella typhimurium and Salmonella choleraesuis var kunzendorf in tissues of experimentally infected swine. Am J Vet Res. 1990;51:619-624.
17. Balthazar EJ, Charles HW, Megibow AJ. Salmonella and Shigellainduced ileitis: CT findings in four patients. J Comput Assist Tomogr. 1996;20:375-378.

18. Chen YH, TP Chen TP, et al. Salmonella Choleraesuis bacteremia in southern Taiwan. Kaohsiung J Med Sci. 1999;15:202-208.

19. Wheeler MD. Endotoxin and Kupffer cell activation in alcoholic liver disease. Alcohol Res Health. 2003;27:300-306.

20. Chen SC, Lee YT, Lai KC, et al. Risk factors for developing metastatic infection from pyogenic liver abscesses. Swiss Med Wkly. 2006;136: 119-126.

21. Holguin F, Moss IM, Brown LAS, Guidot DM. Chronic ethanol ingestion impairs alveolar type II cell glutathione homeostasis and function and predisposes to endotoxin-mediated acute edematous lung injury in rats. $J$ Clin Invest. 1998;101:761-768.

22. Brown LAS, Harris FL, Ping XD, Gauthier TW. Chronic ethanol ingestion and the risk of acute lung injury: a role for glutathione availability? Alcohol. 2004;33:191-197.

23. Arias CA, Contreras GA, Murray BE. Management of multidrug-resistant enterococcal infections. Clin Microbiol Infect. 2010;16:555-562.
International Journal of General Medicine

\section{Publish your work in this journal}

The International Journal of General Medicine is an international, peer-reviewed open-access journal that focuses on general and internal medicine, pathogenesis, epidemiology, diagnosis, monitoring and treatment protocols. The journal is characterized by the rapid reporting of reviews, original research and clinical studies across all disease areas.

\section{Dovepress}

A key focus is the elucidation of disease processes and management protocols resulting in improved outcomes for the patient.The manuscript management system is completely online and includes a very quick and fair peer-review system. Visit http://www.dovepress.com/ testimonials.php to read real quotes from published authors.

Submit your manuscript here: http://www.dovepress.com/international-journal-of-general-medicine-journal 